\title{
Risperidone for children and adolescents with autism spectrum disorder: a systematic review
}

This article was published in the following Dove Press journal:

Neuropsychiatric Disease and Treatment

\section{Narong Maneeton' \\ Benchalak Maneeton' \\ Suwannee Putthisri² \\ Pakapan Woottiluk ${ }^{3}$ \\ Assawin Narkpongphun' \\ Manit Srisurapanont ${ }^{\mid}$ \\ 'Department of Psychiatry, Faculty of Medicine, Chiang Mai University, Chiang Mai, Thailand; ' 2Department of Psychiatry, Faculty of Medicine, Ramathibodi Hospital, Mahidol University, Bangkok, Thailand; ${ }^{3}$ Psychiatric Nursing Division, Faculty of Nursing, Chiang Mai University, Chiang Mai, Thailand}

Correspondence: Narong Maneeton Department of Psychiatry, Faculty of Medicine, Chiang Mai University, I 0 Intawaroros Road, Sriphum, Amphur Muang, Chiang Mai 50200, Thailand

Tel +6653935422

Fax +66 53935426

Email narong.m@cmu.ac.th
Background: Various clinical trials suggested that risperidone was beneficial in the treatment of autism spectrum disorder (ASD) in children and adolescents.

Objective: The aim of this systematic review was to determine the efficacy, acceptability and tolerability of risperidone in the treatment of children and adolescents with ASD.

Data sources: The databases of Scopus, PubMed, CINAHL and Cochrane Controlled Trials Register were searched in February 2017.

Study eligibility criteria, participants and interventions: Eligible RCTs of risperidone in the treatment of child and adolescent patients with ASD. Languages were not restricted.

Study appraisal and synthesis methods: The full-text versions of relevant studies were thoroughly assessed and extracted. The primary efficacy of outcome was the pooled response rate and the pooled mean changed scores of the standardized rating scales for ASD.

Results: A total of 372 randomized subjects from seven RCTs were included in this review. In acute treatment, the pooled mean change score of the Aberrant Behavior Checklist for irritability subscale (ABC-I) and response rate for the risperidone-treated group had a greater significance than that of the placebo-treated group. In the long-term treatment, the pooled mean change score of the CARS in the risperidone-treated group was significantly greater than that in the placebo-treated group. According to the discontinuation phase, the overall pooled relapse rate of the risperidone-treated group was significantly less than that of the placebo-treated group. The rates of pooled overall discontinuation and discontinuation due to adverse events rates were not different between the two groups in acute and long-term treatments.

Limitations: A small study was included in the current review.

Conclusion: In relation to the current systematic review, risperidone is efficacious in the treatment of symptoms in children and adolescents with ASD. Although its acceptability is comparable to placebo, treatment with risperidone is well tolerated in children and adolescents with ASD.

Keywords: Aberrant Behavior Checklist, ABC, Childhood Autism Rating Scale, CARS, efficacy, acceptability, tolerability

\section{Background}

Autism spectrum disorder (ASD) was categorized as a neurodevelopmental disorder according to the Diagnostic and Statistical Manual of Mental Disorders, fifth edition (DSM-5). Its symptoms mainly consist of deficits in social communication and social interaction and restricted, repetitive patterns of behaviors, interests and activities. In addition, several children and adolescents with ASD also present severe behavioral difficulties, including aggression, self-injurious behavior, tantrums and irritability, 
possibly serious enough to interfere with the education and development of children and adolescents as well as the wellbeing of caregivers. ${ }^{1,2}$ Hence, effective treatments for such maladaptive behaviors are necessary.

Current evidence suggests that behavioral therapy possibly reduces aggression and self-injurious behavior and is considered a gold standard therapy for behavioral problems related to ASD. ${ }^{3}$ In addition to individualized responses to behavioral therapy for ASD, the low quality of methodology, limited sample size or short-term treatment is often found in previous studies. ${ }^{3-5}$ Since behavioral therapy involves the use of wide-ranging resources with extensive cost, it may reduce the opportunity to access such intervention for several children with ASD. ${ }^{6}$ Unfortunately, a recent randomized controlled trial (RCT) did not find significant differences between parent training and parent education programs in the management of behavioral problems in children with ASD. ${ }^{7}$

Although a previous RCT reported the effective combined treatment of low-dose risperidone and parent training ${ }^{8}$ and atomoxetine with parent training 9 in children with ASD, its effect size is small. Alternatively, antipsychotics such as haloperidol, risperidone and aripiprazole have efficacy in the treatments of behavioral difficulties associated with ASD. ${ }^{10-13}$

Risperidone, a highly potent blockade of serotonin $2 \mathrm{~A}$ and dopamine D2 receptors, has a promising efficacy in the treatment of both the positive and negative symptoms of schizophrenia. ${ }^{14}$ Furthermore, the evidence from RCTs has illustrated its efficacy in symptomatic treatment for behavioral problems such as irritability, aggression, self-injurious behavior and tantrums in children and adolescents with ASD having the short-term treatment with well tolerability. ${ }^{12,15,16}$ In addition, a previous review supports risperidone as effective in the management of behavioral problems in children with ASD. ${ }^{17,18}$

Since those RCTs have a limited number of participants and the small number of sample size in previous reviews, the current systematic review, which included more studies, is likely to be a method to determine the efficacy and acceptability of risperidone in the treatment of children and adolescents with ASD. Hence, the aim of the current study was to systematically review the efficacy, acceptability and tolerability of risperidone in children and adolescents with ASD.

\section{Subjects and methods Types of studies}

All related RCTs were considered in this review.

\section{Types of participants}

All children and adolescents, age up to 18 years meeting any set of diagnostic criteria of ASD, were included in this review.

\section{Types of interventions}

Risperidone as monotherapy was compared with placebo with no limit to the doses, forms and frequency of treatments. However, if the trial studied several doses, the highest dose was selected for synthesis.

\section{Types of outcome measures}

\section{Primary outcome measures}

The primary outcome measured the mean changed scores of standardized autistic rating scales such as the Aberrant Behavior Checklist (ABC) and Childhood Autism Rating Scale (CARS).

\section{Secondary outcome measures}

The secondary outcomes were as follows:

1. Response rate

2. Relapse rate

3. Discontinuation rates

- Overall discontinuation rate

- Discontinuation rate due to adverse events.

\section{Information sources}

The databases of Scopus, PubMed, CINAHL and Cochrane Controlled Trials Register were searched for relevant articles. According to the publication of risperidone in the PubMed starting in 1988, searches of the publications, therefore, commenced from January 1988 to February 2017. In addition, other databases, including the ClinicalTrials.gov and EU Clinical Trials Register (EU-CTR), were also searched. Searching was limited only to human studies. The references of relevant articles derived from any means were investigated. All RCTs of ASD in children and adolescents were eligible for the current review. Languages of such RCTs were not restricted.

\section{Searches}

To increase the sensitivity for optimally identifying such RCTs, the search technique for the PubMed was confined in the following words and phrases: risperidone AND Autism Spectrum Disorder OR Autistic Disorder OR Pervasive Developmental Disorder OR Asperger syndrome. Similarly, this search technique was utilized for the rest of the databases. 


\section{Study selection}

With regard to the eligible criteria as defined earlier, two reviewers (NM and $\mathrm{BM}$ ) individually scrutinized all the titles and abstracts derived from the electronic databases. After acquiring the full-text version of the relevant articles, two reviewers (NM and BM) independently inspected them. When the disputes between reviewers took place, a conclusion was achieved by consensus.

\section{Data collection process}

Extracting the data from the full-text articles and putting them into the developed extraction form were initially performed by the first reviewer (NM). The second reviewer (BM), then, cautiously rechecked them again. Disagreement between two reviewers was also solved by consensus. When disagreements between two reviewers could not reach a conclusion, the third reviewer (MS)'s judgment was applied.

\section{Data items}

The extracted data accumulated from RCTs comprised the following: 1) related findings used for quality assessments; 2) basic characteristics of participants, diagnostic criteria, study designs and eligible/ineligible criteria; 3) forms, doses and treatment duration of risperidone; 4) important outcomes and 5) intention-to-treat results.

\section{Risk of bias in individual studies}

Assessment of internal validity (quality) for the eligible RCTs was carried out by two reviewers (NM and BM). Based on the Cochrane Collaboration's tool for assessing the risk of bias, the evaluation of such risk of bias consisted of the following: 1) sequence generation (randomization); 2) allocation concealment; 3 ) blinding of participants, personnel and outcomes; 4) incomplete outcome data; 5) selective outcome reporting and 6) other biases. ${ }^{19}$

\section{Summary measures}

The principal outcomes included the efficacy, acceptability and tolerability. The efficacy was determined by either the end point or the mean changed score of a standardized ASD scale and rate of response and relapse for ASD categorized by any set of measures. Based on the previous meta-analysis, acceptability was determined by the rate of overall discontinuation, ${ }^{20}$ and tolerability related to adverse events of the medications ${ }^{21}$ was calculated by the rate of discontinuation owing to such adverse events.

\section{Statistical analyses and synthesis of results}

This systematic review calculated the weighted mean difference (WMD) or a standardized mean difference (SMD) with $95 \% \mathrm{CI}$ and relied on the same or various measurement scales used across studies. If some outcomes, including SD, end point and mean changed scores, were not available, the calculation could be performed by using any of the statistical techniques or by direct substitution. ${ }^{22}$ An inverse variance, weighing the effect of each RCT, was used to calculate the pooled mean end point or the pooled mean changed score with $95 \%$ CIs. ${ }^{19}$

Synthesis of dichotomous outcomes was carried out by calculating the relative risk (RR) with $95 \%$ CI. In the case of the RR precisely being one, it indicates that a difference in results between the intervention and control groups was not revealed. On the contrary, the RR being more or less than one indicates that the treatment, respectively, increases or decreases the risk of the results. The RRs were applied for a comparison of response rates and discontinuation rates between the two groups. The Mantel-Haenszel approach was operated to determine all pooled RRs of discontinuous outcomes with $95 \%$ CIs. ${ }^{19}$ According to the response rate, the number needed to treat (NNT; 95\% CI) was also calculated.

As a rule, the eligible trials in a systematic review are unlikely to be absolutely identical ones, although they are relatively homogeneous. Hence, a random-effects model, speculating that the true effect size varies across the studies, was assigned to synthesize all outcomes in this review. The synthesis of all outcomes was operated by using the RevMan 5.1 (The Nordic Cochrane Centre, The Cochrane Collaboration, Copenhagen, Denmark).

\section{Risk of bias across studies}

If possible, the reporting bias was evaluated by using a funnel plot; a simple scatter plot of the intervention effect estimated from individual studies against a measure of each study's size. If the plot resembles a symmetrical inverted funnel, it could assume the absence of bias. ${ }^{23}$

\section{Test of heterogeneity}

A test of heterogeneity is to examine the similarities of clinical outcomes among the included studies in a systematic review. After such a test was performed in this systematic review, we postulated that the effect size was different since the quality of methodology in each study was varied. The results of each 
trial were tested with regard to whether they were higher and different from the anticipated outcomes by chance alone. Determination of those outcomes was performed by inspecting them as graphical displays as well as applying the test of heterogeneity. Such outcomes were defined as significant heterogeneity when there was an $I^{2}$ of $\geq 50 \%$.

\section{Results}

\section{Study selection}

Based on the search of such databases, there were a total of 746 citations $($ Scopus $=493$, PubMed $=42, \mathrm{CINAHL}=126$, Cochrane Controlled Trials Register $=77$, ClinicalTrials.gov $=5$ and EU-CTR = 3; Figure 1). After the duplicates were removed, 590 citations persisted. After the titles and abstracts from such studies were evaluated, 13 citations still met the inclusion criteria. As a result, the full-text articles of such citations were examined. Six studies were excluded from this review because one studied an adult population, ${ }^{24}$ one had no controlled group, ${ }^{25}$ three were an analysis of secondary outcomes from previous studies ${ }^{26-28}$ and one was an openlabel extension phase of a previous trial. ${ }^{29}$ As a result, a total of seven articles were eligible for this review. ${ }^{12,15,16,29-32}$ However, a relevant or unpublished study fitting the inclusion criteria was not detected.

\section{Study characteristics}

The eligible trials had a study duration of 6-8 weeks for acute treatment, 6 months for long-term treatment and 8 weeks for the withdrawal phase. All participants were randomized to receive risperidone or placebo treatment. The dosage of risperidone was flexible in all trials, except one. ${ }^{31}$ The demographic and clinical characteristics of the risperidone-treated group vs the placebo-treated group were largely well matched across the seven studies. A summary of included RCTs is presented in Table 1.

A total of 372 randomized participants from the included studies were selected for this systematic review. Those participants met the pervasive developmental disorder

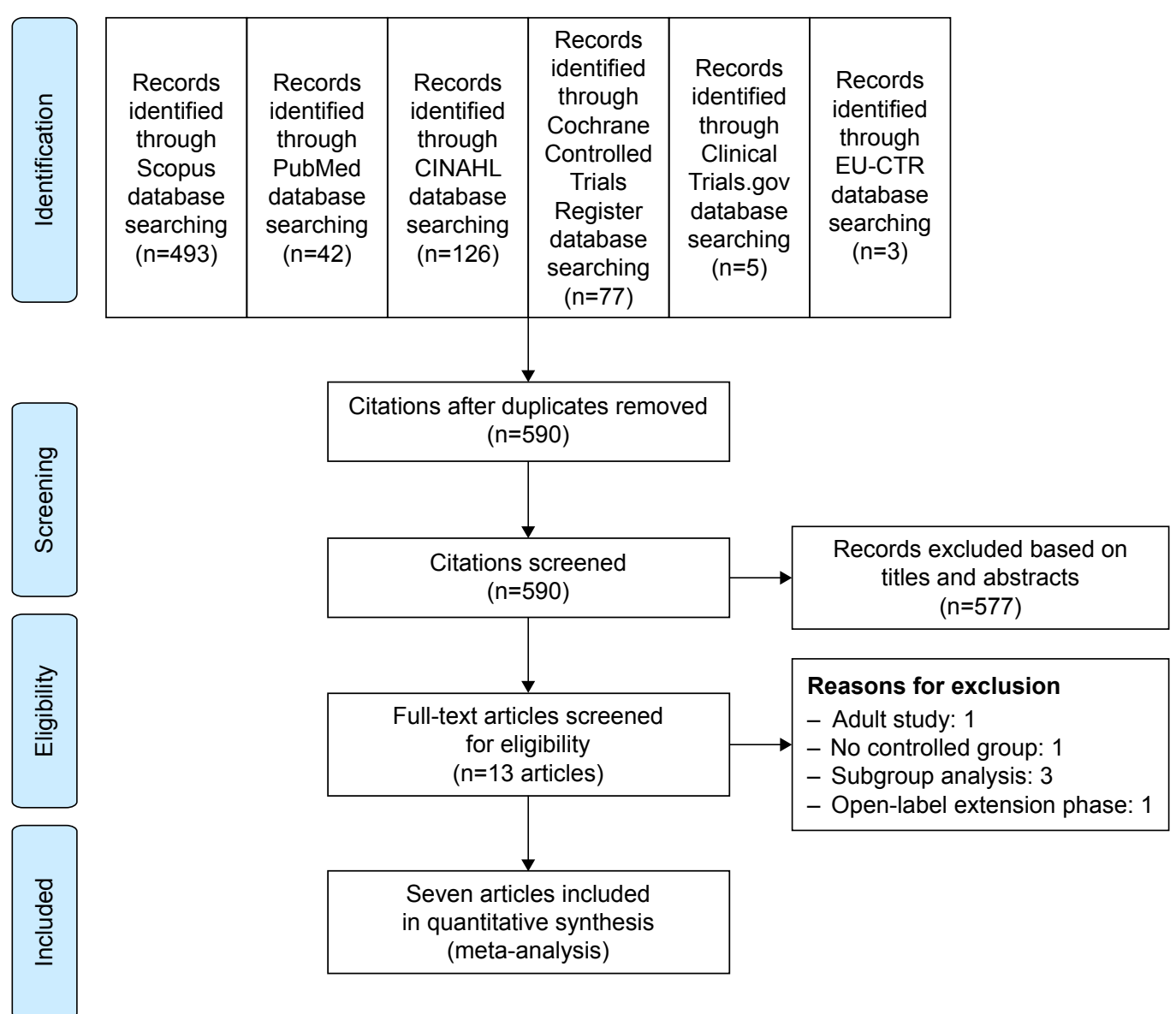

Figure I Flow diagram of the study.

Abbreviation: EU-CTR, EU Clinical Trials Register. 
criteria of the DSM in the fourth edition or fourth edition, text revision. The ages of the participants were up to 17 years. The participant was individually randomized to receive risperidone or placebo. The basic characteristics of the eligible studies are summarized in Table 1.

Since the ABC was applied in all acute treatment and withdrawal studies, the CARS was used in all longterm treatment studies; the WMDs of the mean changed scores could be applied for the estimation and synthesis of the outcomes. Although the response rates have been reported only in acute treatment studies, discontinuation rates have been shown in both short- and long-term studies. All included withdrawal studies displayed the relapse rate measured by the $\mathrm{ABC}$ for irritability subscale (ABC-I) and the Clinical Global Impression of Improvement (CGI-I) or Clinical Global Impression of Symptom Change (CGI-SC).

\section{Risk of bias within studies}

Most studies have addressed the generated sequence for randomization, allocation concealment, blinding techniques for personnel and outcomes, incomplete outcome data and other biases. The intention-to-treat analysis was used in all studies, except one trial. ${ }^{31}$ The summary of bias is shown in Figure 2.

\section{Synthesis of results \\ Efficacy}

Acute treatment

The heterogeneity was significantly found in the pooled response rate and mean changed scores of ABC-I. The overall pooled response rate of the risperidone-treated group had a greater significance than that of the placebo-treated group with a RR $(95 \% \mathrm{CI})$ of $2.57(1.35,4.86), I^{2}=77 \%$ (Figure 3). Based on the pooled response rate, the NNT $(95 \% \mathrm{CI})$ was $2.3(1.8,3.0)$. The pooled mean changed scores in the risperidone-treated group for $\mathrm{ABC}-\mathrm{I}$ (Figure 4), ABC-lethargy/social withdrawal (SW), ABC-stereotypic behavior $(\mathrm{S}), \mathrm{ABC}$-hyperactivity/noncompliance $(\mathrm{H})$ and ABC-inappropriate speech (IS) were also significantly higher than those in the placebo-treated group with WMDs $(95 \%$ CI) of $-8.50(-11.99,-5.00), I^{2}=62 \% ;-3.11(-5.28,-0.94)$, $I^{2}=0 \% ;-2.58(-3.74,-1.41), I^{2}=3 \% ;-8.73(-11.44,-6.02)$, $I^{2}=0 \%$ and $-1.08(-2.06,-0.10), I^{2}=0 \%$, respectively.

\section{Long-term treatment}

The heterogeneity was significantly noted in the WMD of the pooled mean changed scores of the CARS. The pooled mean changed score of the CARS in the risperidone-treated group was significantly greater than that in the placebotreated group (WMD [95\% CI] of $-4.62[-7.84,-1.40]$, $I^{2}=78 \%$; Figure 5).

\section{Discontinuation study outcomes}

The heterogeneity was not significantly different in the pooled relapse rate. The overall pooled relapse rate of the risperidone-treated group was significantly less than that of the placebo-treated group with a RR (95\% CI) of $0.30(0.13,0.68), I^{2}=0 \%$. Based on a discontinuation study, a mean changed score of ABC-I in the placebotreated group significantly increased more than that in the risperidone-treated group at the end of the study (Figure 6). Unfortunately, mean changed scores of other ABC subscales were not significantly different between the two groups. ${ }^{32}$

\section{Main adverse event}

The pooled mean changed weight scores in the risperidonetreated group had a greater significance than those in the placebo-treated group in both short- and long-term treatments with WMDs $(95 \% \mathrm{CI})$ of $1.75(1.25,2.25), I^{2}=0 \%$ and 1.57 $(0.38,2.76), P^{2}=37 \%$, respectively. The increased appetite rate in the risperidone-treated group was also significantly higher than that in the placebo-treated group in short-term treatment with a RR $(95 \% \mathrm{CI})$ of $2.64(1.76,3.96), I^{2}=0 \%$. The other adverse events more commonly reported in the risperidonetreated group included the following: drowsiness, ${ }^{12,16,30,31}$ somnolence, ${ }^{15,16}$ fatigue, ${ }^{12,15,32}$ anxiety, ${ }^{32}$ hypersalivation, ${ }^{12,31}$ and elevation of prolactin level. ${ }^{16,31}$ However, serious adverse events were not reported.

\section{Overall discontinuation rate (acceptability)}

According to the overall discontinuation rate, a significance of heterogeneity was not observed in acute treatment. The pooled overall discontinuation rates were not different between two groups in acute and long-term treatments with RRs $\left(95 \%\right.$ CI) of $0.41(0.14,1.14), I^{2}=55 \%$, and $1.13(0.17$, 7.47), $I^{2}=0 \%$, respectively.

\section{Discontinuation rate due to adverse events (tolerability)}

No significance of heterogeneity was found in the discontinuation rate due to adverse events. The pooled discontinuation rates due to adverse events between two groups in both acute 


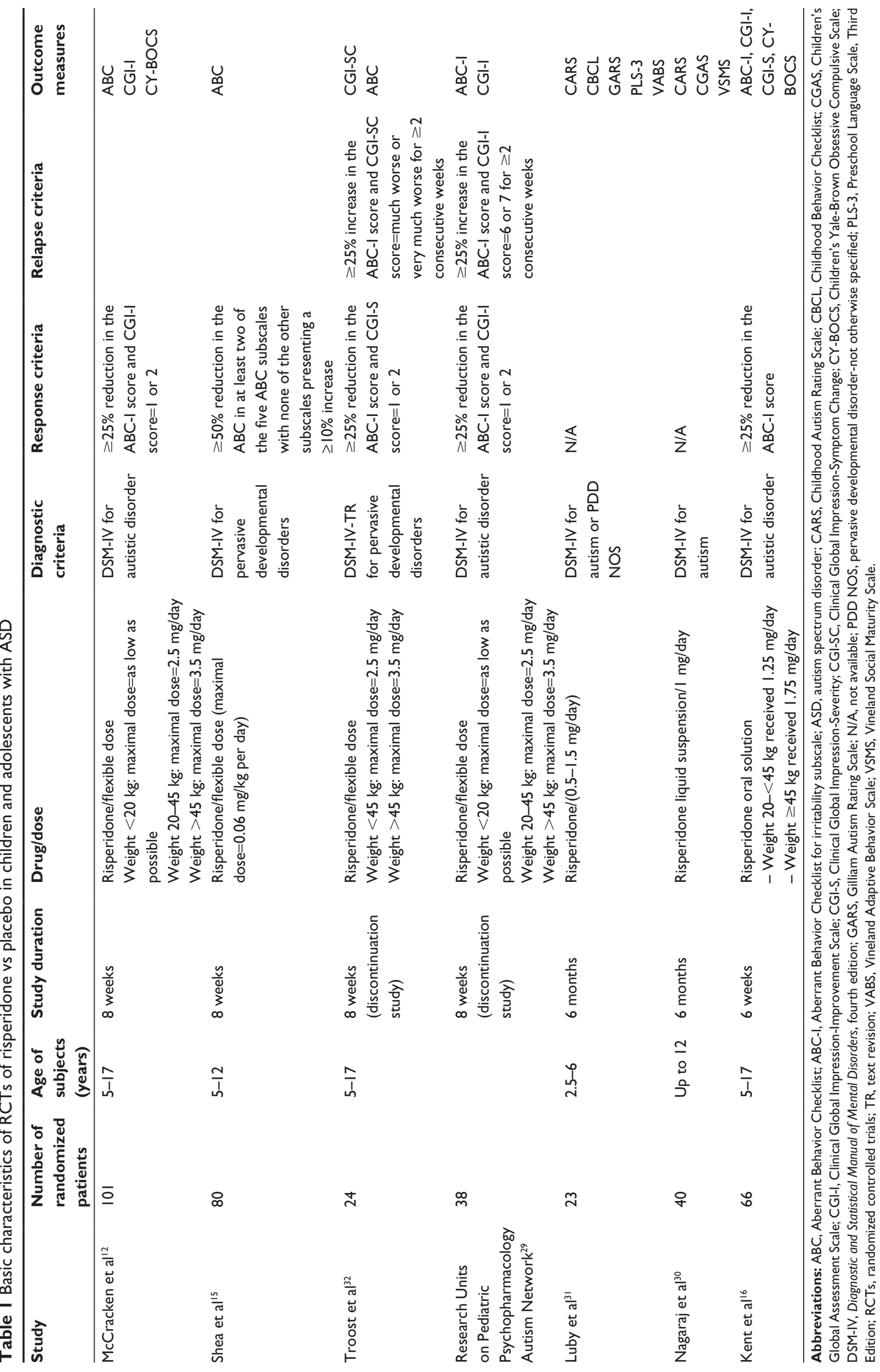




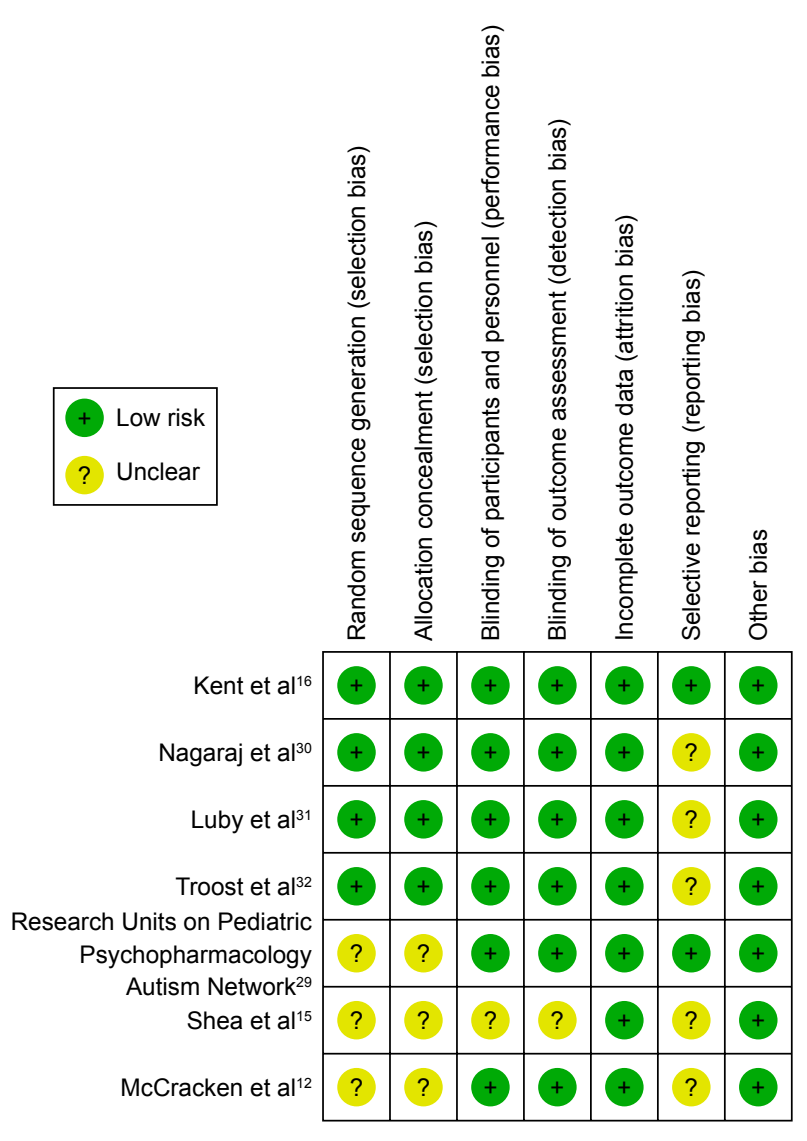

Figure 2 Summary of risk of bias in RCTs of risperidone vs placebo in children and adolescents with ASD.

Abbreviations: ASD, autism spectrum disorder; RCTs, randomized controlled trials.

and long-term treatments had no difference with RRs (95\% CI) of $1.05(0.18,5.93), I^{2}=0 \%$, and $1.10(0.17,7.28), I^{2}=0 \%$, respectively.

\section{Risk of bias across studies}

If a systematic review gathers $<10$ studies, a funnel plot evaluating the publication bias may not have sufficient power to determine the chances of real asymmetry occurring due to the included results. ${ }^{23}$ As a result, the test of funnel plot was not accomplished because this review contained only seven RCTs.

\section{Discussion}

The findings of the current review has a promising result that risperidone is efficacious in the treatment of symptoms of ASD in short-term, long-term and withdrawal phases. The overall response rate was $70 \%$. In relation to the response rate, the NNT of three suggests that one in every three ASD children and adolescents has benefits from treatment with risperidone. However, treatment with risperidone increased the incidence of adverse events, particularly in weight gain. Unfortunately, the acceptability of treatment with risperidone in such patients was comparable to placebo. However, tolerability of treatment with risperidone was not different from placebo.

Several studies of antipsychotics for the treatment of ASD have been conducted. For instance, a previous study found that haloperidol could reduce the behavioral symptoms and improve discrimination learning in autistic children. ${ }^{10,11,33}$ Recent evidence has indicated that treatment with risperidone is more efficacious and tolerable than haloperidol in the reduction in behavioral symptoms and impulsivity and improvement in language skills, sensory motor and impaired social relations in both the short and long terms. ${ }^{34,35}$ However, recent evidence indicated that the efficacy and safety of risperidone and aripiprazole in the treatment of ASD children and adolescents were comparable. ${ }^{36}$ Thus, both risperidone and aripiprazole, except haloperidol, can be alternatively chosen to treat such children and adolescents possibly based on their clinical profiles and patient's preference. ${ }^{36}$

The previous studies suggest that both the first- and second-generation antipsychotics in the treatment of ASD children and adolescents have shown safety. ${ }^{37,38}$ The firstgeneration antipsychotics increased the risk of extrapyramidal side effects, while the second-generation agents have a greater adverse event in weight gain and health problems in both short- and long-term studies. ${ }^{31,32,38}$ The evidence from the current review has supported those previous studies.

In this review, the acceptability of risperidone, measured by the overall discontinuation rate, was not better

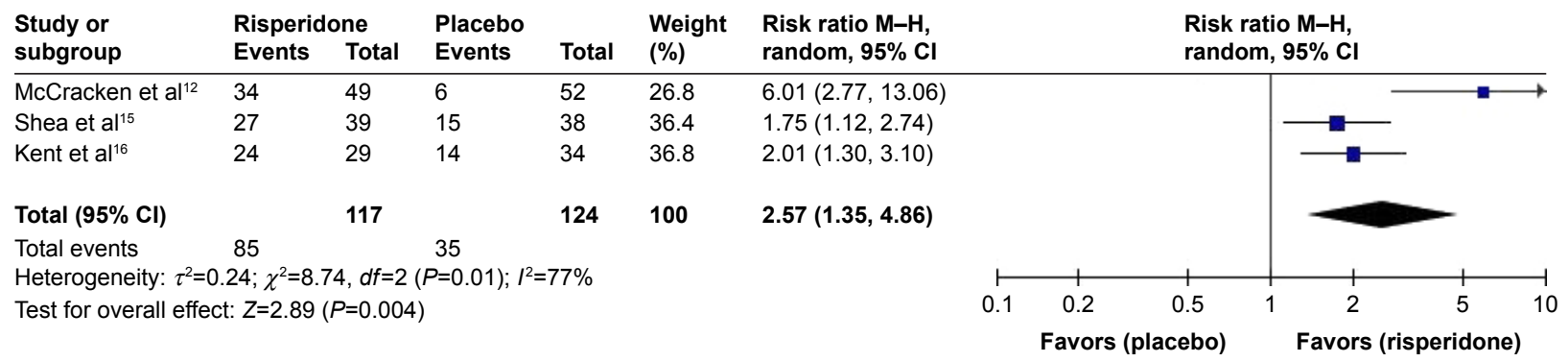

Figure 3 Forest plot of comparison of RR $(95 \% \mathrm{Cl})$ for clinical response rates of risperidone vs placebo in children and adolescents with ASD in acute treatment. Abbreviations: ASD, autism spectrum disorder; $d f$, degrees of freedom; $M-H$, Mantel-Haenszel; RR, relative risk. 


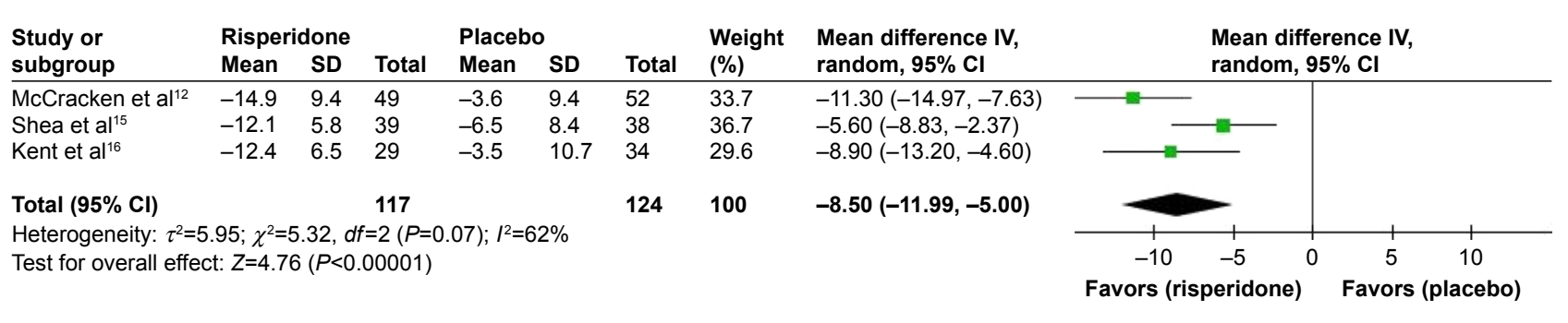

Figure 4 Forest plot of comparison of mean change scores from baseline of $A B C-I$ scores $(95 \% \mathrm{Cl})$ of risperidone vs placebo in children and adolescents with ASD in acute treatment.

Abbreviations: ABC-I, Aberrant Behavior Checklist for irritability subscale; ASD, autism spectrum disorder; df, degrees of freedom; IV, inverse variance.

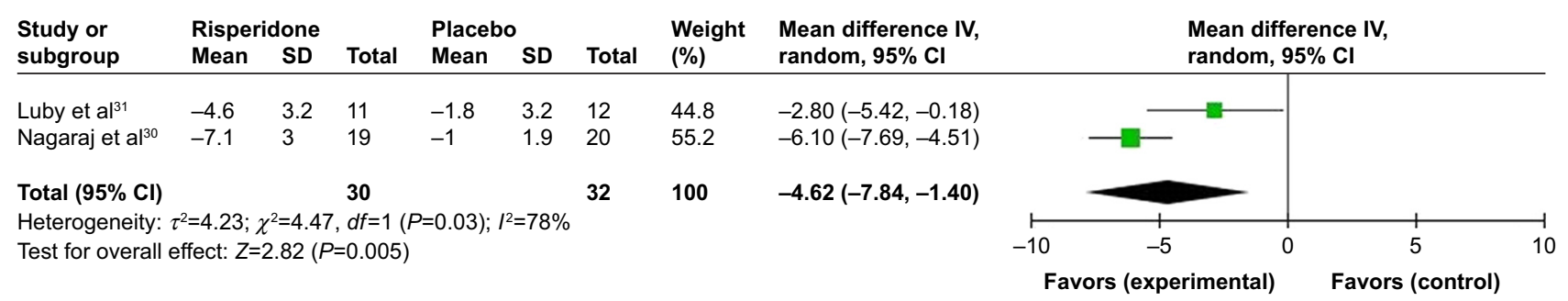

Figure 5 Forest plot of comparison of mean change scores from baseline of CARS scores $(95 \% \mathrm{Cl})$ of risperidone vs placebo in children and adolescents with ASD in longterm treatment.

Abbreviations: ASD, autism spectrum disorder; CARS, Childhood Autism Rating Scale; $d f$, degrees of freedom; IV, inverse variance.

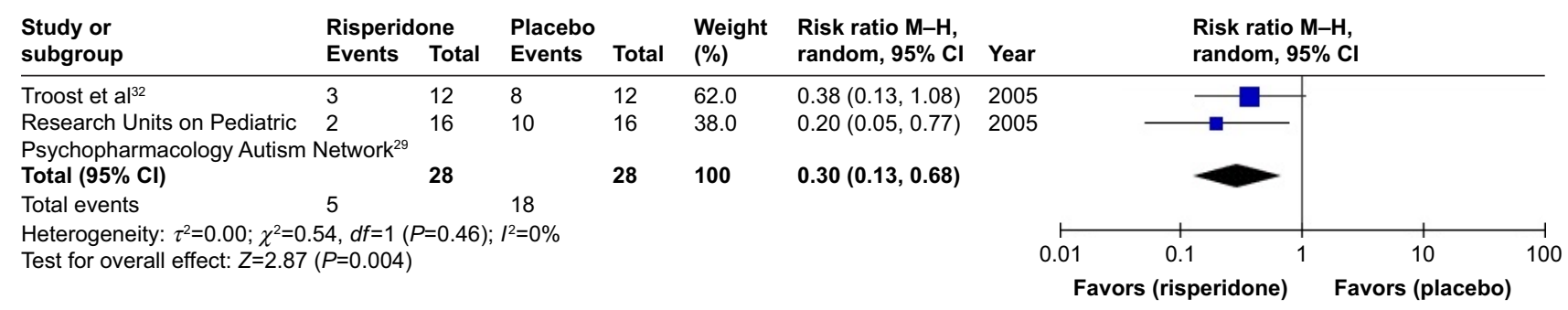

Figure 6 Forest plot of comparison of RR $(95 \% \mathrm{Cl})$ for a relapse rate of risperidone vs placebo in children and adolescents with ASD in discontinuation treatment. Abbreviations: ASD, autism spectrum disorder; $d f$, degrees of freedom; M-H, Mantel-Haenszel; RR, relative risk.

than that of the placebo. Similarly, the tolerability of risperidone, measured by the discontinuation rate due to adverse events, was also comparable to the placebo which was compatible with the previous study of aripiprazole. ${ }^{13}$ Although risperidone in ASD children and adolescents is well tolerated, its acceptability is comparable to placebo; the use of risperidone in such patients, therefore, should be carefully considered.

There were some limitations in this review. First, this review included a small $\mathrm{RCT}^{31}$ into quantitative synthesis which may not have the power to determine the effect of intervention. ${ }^{39}$ In addition, small RCTs potentially illustrate a greater effect than larger RCTs. ${ }^{40}$ As a result, such RCTs may be limited for evaluation regarding scientific outcomes and may reduce the power of the current study. Second, we found that some eligible studies received financial support by a patent-holding company for risperidone. ${ }^{15,16,31}$
Although the included studies were RCTs, ${ }^{15,16,31}$ interpretation of those outcomes in this review should be considered cautiously. Finally, based on the risk of bias assessment for eligible studies, there were some potential bias concerns, especially in reporting and selection. In addition, the test of a funnel plot to define asymmetry could not be performed due to limited included trials. ${ }^{23}$ Subsequently, publication bias may not be excluded.

\section{Conclusion}

With regard to the limited evidence from the current systematic review, risperidone is efficacious in the treatment of symptoms in children and adolescents with ASD. Although its acceptability is comparable, children and adolescents tolerate treatment with risperidone as well. Due to a limited sample size, further well-defined and large sample size studies should be carried out to validate those findings. 


\section{Acknowledgments}

The authors would like to thank Ms Ruth Barnard Leatherman for the manuscript editing. Narong Maneeton and Benchalak Maneeton are joint first authors.

\section{Author contributions}

All authors made substantial contributions to conception and design, acquisition of data, or analysis and interpretation of data; took part in drafting the article or revising it critically for important intellectual content; gave final approval of the version to be published; and agree to be accountable for all aspects of the work.

\section{Disclosure}

NM received travel reimbursement from Lundbeck and Pfizer. BM received honoraria and/or travel reimbursement from Lundbeck, Pfizer and Servier. MS received honoraria from Lundbeck and Sumitomo Dainippon Pharma. The authors report no other conflicts of interest in this work.

\section{References}

1. Hill AP, Zuckerman KE, Hagen AD, et al. Aggressive behavior problems in children with autism spectrum disorders: prevalence and correlates in a large clinical sample. Res Autism Spectr Disord. 2014; 8(9):1121-1133.

2. Soke GN, Rosenberg SA, Hamman RF, et al. Brief report: prevalence of self-injurious behaviors among children with autism spectrum disorder - a population-based study. J Autism Dev Disord. 2016;46(11): 3607-3614.

3. Schreibman L. Intensive behavioral/psychoeducational treatments for autism: research needs and future directions. J Autism Dev Disord. 2000;30(5):373-378

4. Ospina MB, Krebs Seida J, Clark B, et al. Behavioural and developmental interventions for autism spectrum disorder: a clinical systematic review. PLoS One. 2008;3(11):e3755.

5. Spreckley M, Boyd R. Efficacy of applied behavioral intervention in preschool children with autism for improving cognitive, language, and adaptive behavior: a systematic review and meta-analysis. $J$ Pediatr. 2009;154(3):338-344.

6. Masi A, DeMayo MM, Glozier N, Guastella AJ. An overview of autism spectrum disorder, heterogeneity and treatment options. Neurosci Bull. 2017;33(2):183-193.

7. Bearss K, Johnson C, Smith T, et al. Effect of parent training vs parent education on behavioral problems in children with autism spectrum disorder: a randomized clinical trial. JAMA. 2015;313(15):1524-1533.

8. Aman MG, McDougle CJ, Scahill L, et al. Medication and parent training in children with pervasive developmental disorders and serious behavior problems: results from a randomized clinical trial. J Am Acad Child Adolesc Psychiatry. 2009;48(12):1143-1154.

9. Handen BL, Aman MG, Arnold LE, et al. Atomoxetine, parent training, and their combination in children with autism spectrum disorder and attention-deficit/hyperactivity disorder. J Am Acad Child Adolesc Psychiatry. 2015;54(11):905-915.

10. Anderson LT, Campbell M, Grega DM, Perry R, Small AM, Green WH. Haloperidol in the treatment of infantile autism: effects on learning and behavioral symptoms. Am J Psychiatry. 1984;141(10):1195-1202.

11. Anderson LT, Campbell M, Adams P, Small AM, Perry R, Shell J. The effects of haloperidol on discrimination learning and behavioral symptoms in autistic children. J Autism Dev Disord. 1989;19(2):227-239.
12. McCracken JT, McGough J, Shah B, et al; Research Units on Pediatric Psychopharmacology Autism Network. Risperidone in children with autism and serious behavioral problems. $N$ Engl J Med. 2002;347(5):314-321.

13. Marcus RN, Owen R, Kamen L, et al. A placebo-controlled, fixeddose study of aripiprazole in children and adolescents with irritability associated with autistic disorder. J Am Acad Child Adolesc Psychiatry. 2009;48(11):1110-1119.

14. Marder SR, Meibach RC. Risperidone in the treatment of schizophrenia. Am J Psychiatry. 1994;151(6):825-835.

15. Shea S, Turgay A, Carroll A, et al. Risperidone in the treatment of disruptive behavioral symptoms in children with autistic and other pervasive developmental disorders. Pediatrics. 2004;114(5):e634-e641.

16. Kent JM, Kushner S, Ning X, et al. Risperidone dosing in children and adolescents with autistic disorder: a double-blind, placebo-controlled study. J Autism Dev Disord. 2013;43(8):1773-1783.

17. Sharma A, Shaw SR. Efficacy of risperidone in managing maladaptive behaviors for children with autistic spectrum disorder: a meta-analysis. J Pediatr Health Care. 2012;26(4):291-299.

18. Williamson E, Sathe NA, Andrews JC, et al. Medical Therapies for Children with Autism Spectrum Disorder - An Update. Rockville, MD: Agency for Healthcare Research and Quality (US); 2017.

19. Higgins JPT, Altman DG. Assessing risk of bias in included studies. In: Higgins JPT, Green S, editors. Cochrane Handbook for Systematic Reviews of Interventions. London: The Cochrane Collaboration; 2011. Version 5.1.0.

20. Cipriani A, Furukawa TA, Salanti G, et al. Comparative efficacy and acceptability of 12 new-generation antidepressants: a multipletreatments meta-analysis. Lancet. 2009;373(9665):746-758.

21. Papakostas GI. Tolerability of modern antidepressants. $J$ Clin Psychiatry. 2008;69(Suppl E1):8-13.

22. Wiebe N, Vandermeer B, Platt RW, Klassen TP, Moher D, Barrowman NJ. A systematic review identifies a lack of standardization in methods for handling missing variance data. J Clin Epidemiol. 2006;59(4): 342-353.

23. Sterne JAC, Egger M, Moher D. Addressing reporting biases. In: Higgins JPT, Green S, editors. Cochrane Handbook for Systematic Reviews of Interventions. London: The Cochrane Collaboration; 2011. Version 5.1.0.

24. McDougle CJ, Holmes JP, Carlson DC, Pelton GH, Cohen DJ, Price LH. A double-blind, placebo-controlled study of risperidone in adults with autistic disorder and other pervasive developmental disorders. Arch Gen Psychiatry. 1998;55(7):633-641.

25. Gagliano A, Germano E, Pustorino G, et al. Risperidone treatment of children with autistic disorder: effectiveness, tolerability, and pharmacokinetic implications. J Child Adolesc Psychopharmacol. 2004;14(1): 39-47.

26. McDougle CJ, Scahill L, Aman MG, et al. Risperidone for the core symptom domains of autism: results from the study by the autism network of the research units on pediatric psychopharmacology. Am J Psychiatry. 2005;162(6):1142-1148.

27. Pandina GJ, Bossie CA, Youssef E, Zhu Y, Dunbar F. Risperidone improves behavioral symptoms in children with autism in a randomized, double-blind, placebo-controlled trial. J Autism Dev Disord. 2007;37(2): 367-373.

28. Aman MG, Hollway JA, McDougle CJ, et al. Cognitive effects of risperidone in children with autism and irritable behavior. J Child Adolesc Psychopharmacol. 2008;18(3):227-236.

29. Research Units on Pediatric Psychopharmacology Autism Network. Risperidone treatment of autistic disorder: longer-term benefits and blinded discontinuation after 6 months. Am J Psychiatry. 2005;162(7): 1361-1369.

30. Nagaraj R, Singhi P, Malhi P. Risperidone in children with autism: randomized, placebo-controlled, double-blind study. J Child Neurol. 2006;21(6):450-455.

31. Luby J, Mrakotsky C, Stalets MM, et al. Risperidone in preschool children with autistic spectrum disorders: an investigation of safety and efficacy. J Child Adolesc Psychopharmacol. 2006;16(5):575-587. 
32. Troost PW, Lahuis BE, Steenhuis MP, et al. Long-term effects of risperidone in children with autism spectrum disorders: a placebo discontinuation study. J Am Acad Child Adolesc Psychiatry. 2005;44(11): 1137-1144.

33. Campbell M, Anderson LT, Small AM, Perry R, Green WH, Caplan R. The effects of haloperidol on learning and behavior in autistic children. J Autism Dev Disord. 1982;12(2):167-175.

34. Miral S, Gencer O, Inal-Emiroglu FN, Baykara B, Baykara A, Dirik E. Risperidone versus haloperidol in children and adolescents with AD: a randomized, controlled, double-blind trial. Eur Child Adolesc Psychiatry. 2008;17(1):1-8.

35. Gencer O, Emiroglu FN, Miral S, Baykara B, Baykara A, Dirik E. Comparison of long-term efficacy and safety of risperidone and haloperidol in children and adolescents with autistic disorder. An open label maintenance study. Eur Child Adolesc Psychiatry. 2008;17(4):217-225.

36. Ghanizadeh A, Sahraeizadeh A, Berk M. A head-to-head comparison of aripiprazole and risperidone for safety and treating autistic disorders, a randomized double blind clinical trial. Child Psychiatry Hum Dev. 2014;45(2):185-192.
37. Campbell M, Armenteros JL, Malone RP, Adams PB, Eisenberg ZW, Overall JE. Neuroleptic-related dyskinesias in autistic children: a prospective, longitudinal study. J Am Acad Child Adolesc Psychiatry. 1997;36(6): 835-843.

38. Malone RP, Waheed A. The role of antipsychotics in the management of behavioural symptoms in children and adolescents with autism. Drugs. 2009;69(5):535-548.

39. Turner RM, Bird SM, Higgins JP. The impact of study size on metaanalyses: examination of underpowered studies in Cochrane reviews. PLoS One. 2013;8(3):e59202.

40. Sterne JA, Gavaghan D, Egger M. Publication and related bias in metaanalysis: power of statistical tests and prevalence in the literature. J Clin Epidemiol. 2000;53(11):1119-1129.

\section{Publish your work in this journal}

Neuropsychiatric Disease and Treatment is an international, peerreviewed journal of clinical therapeutics and pharmacology focusing on concise rapid reporting of clinical or pre-clinical studies on a range of neuropsychiatric and neurological disorders. This journal is indexed on PubMed Central, the 'PsycINFO' database and CAS, and is the official journal of The International Neuropsychiatric Association (INA). The manuscript management system is completely online and includes a very quick and fair peer-review system, which is all easy to use. Visit http://www.dovepress.com/testimonials.php to read real quotes from published authors. 